\title{
A SURGICAL SURPRISE: A RARE CASE REPORT
}

Jaya Shree V1, Rajalakshmi M²

\section{HOW TO CITE THIS ARTICLE:}

Jaya Shree V, Rajalakshmi M. "A Surgical Surprise: A Rare Case Report". Journal of Evolution of Medical and Dental Sciences 2014; Vol. 3, Issue 35, August 14; Page: 9185-9187, DOI: 10.14260/jemds/2014/3185

ABSTRACT: Implantation of the blastocyst anywhere else other than the uterine cavity is ectopic pregnancy. Ovarian Pregnancy is one of the rare forms of an ectopic pregnancy. The incidence of ovarian pregnancy is 0.15 to $3 \%{ }^{1}$ of all ectopic pregnancies. Here we present a case of ruptured ovarian pregnancy, which was preoperatively diagnosed as ruptured tubal pregnancy and later found to be an ovarian pregnancy at laparotomy.

KEYWORDS: Multigravida, Primary ovarian pregnancy, Partial ovariectomy.

INTRODUCTION: Primary ectopic pregnancy is one of the rarest varieties of ectopic pregnancy and incidence varies from 1 in 7000 to 1 in $40000^{2,3}$ pregnancies. The primary difference between a tubal ectopic pregnancy and an ovarian pregnancy is that it is not associated with PID or infertility. The risk of ovarian ectopic pregnancy in patients using IUDs is controversial.

CASE REPORT: Mrs. X, A 34 year old female, Gravida: Three, Para: one, Abortion: One, reported to the gynecological casualty with 46 days of amenorrhea, abdominal pain and giddiness for two hours. Her prior menstrual cycles were normal. Urine beta hCG pregnancy test was positive.

On examination, patient was conscious; pallor was present, with a pulse rate of $90 /$ minute, blood pressure of $90 / 70 \mathrm{~mm} \mathrm{Hg}$ and tenderness in the right iliac fossa. A brownish discharge was noticed on speculum examination.

Bimanual examination showed normal uterine size, cervical movement tenderness and fullness of the fornices. Pelvic ultrasonography revealed a right adnexal mass with empty uterine cavity, thickened endometrium and free fluid in pouch of Douglas.

A provisional diagnosis of a ruptured ectopic pregnancy was considered and emergency laparotomy was performed. Intraoperatively hemoperitoneum containing blood clots (approximately 300 - 400 grams) and blood (approximately 500ml) was found. Uterus, bilateral tubes and left ovary were normal with no signs of tubal abortion.

The right ovary was enlarged with bleeding from the surface of the ovary. Partial ovariectomy was done including the bleeding site and sent for histopathological examination with a differential diagnosis of hemorrhagic corpus luteal cyst or ruptured ovarian pregnancy. Intraoperatively one unit of blood was transfused. Her postoperative period was uneventful. 


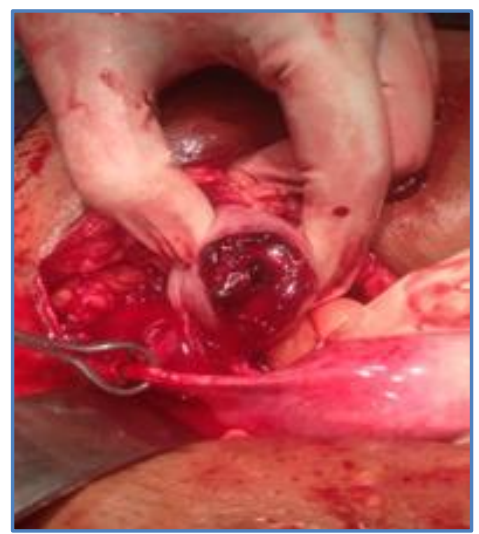

\section{Figure 1: Intraoperative findings of} ruptured right ovarian pregnancy

Histopathological examination showed chorionic villi and trophoblastic tissue in a background of hemorrhagic ovarian stroma.

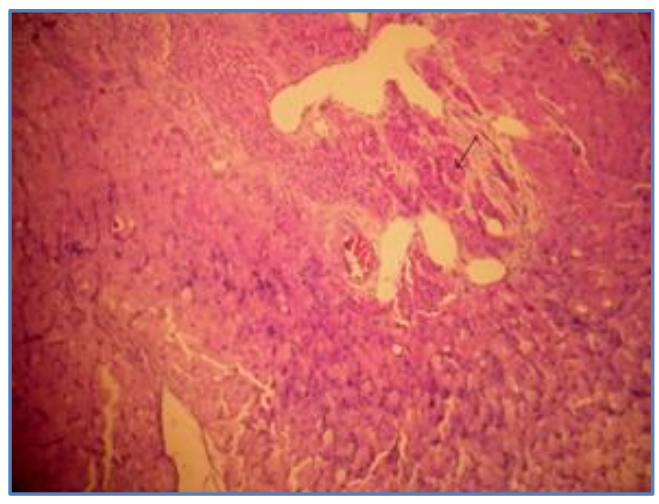

\section{Figure 2: Microscopic image showing trophoblastic tissue In ovarian stroma. (H \& E staining $100 X$ magnification)}

DISCUSSION: The ovarian pregnancy may be misdiagnosed as a corpus luteum cyst. Hence the primary criteria for the diagnosis of ovarian pregnancy were described by Spiegelberg 4 in 1878.

The fallopian tube on the affected side must be intact.

The fetal sac must occupy the position of the ovary.

The ovary must be connected to the uterus by the ovarian ligament.

Ovarian tissue must be located in the sac wall.

Ultrasound, especially TVS has proved to be an invaluable tool in the diagnosis, where hyperechoic appearance of the trophoblast surrounded by thickened hypoechoic ovarian tissue is the only indication of an ovarian ectopic gestation. ${ }^{5}$ Even then; it can be mistaken for a hemorrhagic corpus luteum or ovarian cyst.

In the past, ovarian pregnancy has been treated by ipsilateral ovariectomy, but the trend has shifted towards conservative surgery like partial ovariectomy or cystectomy. The use of methotrexate in a carefully selected case may further be beneficial in conserving future fertility. ${ }^{6}$ 
Medical treatment has a limited role as diagnosis of ovarian ectopic may be difficult until we perform laparoscopy. Laparoscopy is the gold standard for diagnosis and treatment of ovarian ectopic pregnancy. ${ }^{7}$

\section{REFERENCES:}

1. Itil IM, Ozcan 0, et al. Primary ovarian pregnancy: a case report and review of the literature. Ege Tip Dergisi 2004; 43 (2):113-5.

2. Hallatt JG. Primary ovarian pregnancy: a report of twenty-five cases. Am J Obstet Gynecol 1982; 143: 55-60.

3. Grimes HG, Nosal RA, Gallagher JC. Ovarian pregnancy: a series of 24 cases. Obstet Gynecol 1983; 61: 174-180.

4. Spigelberg 0. Casusistik der ovarial schwangerschaft. Arch Gynaecol 1878; 13: 73.

5. Bouyer J, Coste J, Fernandez H et al. Sites of ectopic pregnancy: A 10 year population-based study of 1800 cases. Human Reproduction 2002; 17 (12): 3224-3230.

6. Raziel A, Golan A. Primary ovarian pregnancy successfully treated with methotrexate. Am J Obstet Gynecol 1993; 158: 1362.

7. Seinera P, Di Gregorio A, Arisio R, Decko A, Crana F. Ovarian pregnancy and operative laparoscopy: report of eight cases. Hum Reprod. 1997 Mar; 12 (3): 608-10.

\section{AUTHORS:}

1. Jaya Shree V.

2. Rajalakshmi M.

\section{PARTICULARS OF CONTRIBUTORS:}

1. Associate Professor, Department of Obstetrics and Gynaecology, Rajah Muthiah Medical College.

2. Post Graduate Student, Department of Obstetrics and Gynaecology, Rajah Muthiah Medical College.

\section{NAME ADDRESS EMAIL ID OF THE CORRESPONDING AUTHOR:}

Dr. Jayashree $V$,

E-2, New Kothangudi Quarters,

Annamalai Nagar,

Chidambaram-608002.

Email: shree_ram78@yahoo.co.in

drramyasaravana@gmail.com

Date of Submission: 22/07/2014.

Date of Peer Review: 23/07/2014.

Date of Acceptance: 06/08/2014.

Date of Publishing: 12/08/2014. 